\title{
Processus de gentrification et demande sociale citadine. Exemple du centre-ville de Strasbourg
}

Process of gentrification and urban social demand. The example of the centre of Strasbourg

Der Prozess der Gentrification und die sozial bedingte städtische Nachfrage.

Beispiel des Stadtzntrums von Strassburg

\section{Philippe Gerber}

\section{OpenEdition}

\section{Journals}

Édition électronique

URL : https://journals.openedition.org/rge/4430

DOI : $10.4000 /$ rge.4430

ISSN : 2108-6478

\section{Éditeur}

Association des géographes de l'Est

Édition imprimée

Date de publication : 1 avril 1999

ISSN : 0035-3213

\section{Référence électronique}

Philippe Gerber, «Processus de gentrification et demande sociale citadine. Exemple du centre-ville de Strasbourg ", Revue Géographique de l'Est [En ligne], vol. 39 / 2-3 | 1999, mis en ligne le 05 septembre 2013, consulté le 21 septembre 2021. URL : http://journals.openedition.org/rge/4430 ; DOI : https:// doi.org/10.4000/rge.4430

Ce document a été généré automatiquement le 21 septembre 2021

Tous droits réservés 


\title{
Processus de gentrification et demande sociale citadine. Exemple du centre-ville de Strasbourg
}

\author{
Process of gentrification and urban social demand. The example of the centre of \\ Strasbourg \\ Der Prozess der Gentrification und die sozial bedingte städtische Nachfrage. \\ Beispiel des Stadtzntrums von Strassburg
}

Philippe Gerber

1 La ville et ses mutations internes supposent une connaissance et une recherche géographiques sans cesse approfondies. L'écologie urbaine factorielle propose l'examen, dans cet espace, de l'évolution des rapports entre les structures sociales (classes d'âge, groupes socio-professionnels...) et l'organisation territoriale des groupes humains. Les analyses factorielles, méthodes qui se greffent sur l'explication de la répartition socio-spatiale de la ville, permettent de caractériser cette distribution grâce à des filtres communs, mais qui supposent bien entendu, selon le cas, des résidus par rapport aux modèles intra-urbains. La gentrification fait partie d'un processus de l'écologie urbaine puisqu'il s'agit d'une reconstitution sociale d'une aire urbaine spécifique et importante, à savoir le centre d'une ville. Employé pour la première fois dans les années soixante à propos de la capitale britannique ${ }^{1}$, le terme de gentrification ne figure pas encore dans les dictionnaires usuels français. Il trouve par contre sa place au sein de lexiques spécialisés ${ }^{2}$, avec des définitions nombreuses et variées. Après leur étude approfondie ${ }^{3}$, nous retiendrons la suivante : «la gentrification est un processus de recentralisation spatiale et de revitalisation éco-sociologique des centres anciens de villes; il se concrétise par une "infiltration" de classes moyennes et/ou aisées remplaçant progressivement des catégories sociales défavorisées ». Un phénomène de restructuration socio-résidentielle se développe ainsi dans les centres de villes car il participe in fine à un autre processus, plus global, celui de la recentralisation urbaine qui suppose, conjointement, des mutations fonctionnelles économiques, administratives et commerciales. En outre, la gentrification est hétérogène aux échelles 
inter- et intra-urbaines; elle ne se manifeste ni systématiquement dans toutes les agglomérations - même si elle touche de nombreux pays ${ }^{4}-$, ni uniformément dans les rues d'un cœur urbain.

Divers facteurs ont été avancés par des chercheurs ${ }^{5}$ pour comprendre ce processus. En amont, certaines politiques de logement nationales et/ou locales influencent le marché de l'immobilier qui, lui, entretient des rapports étroits entre offre et demande. Une combinaison de ces rapports s'opère pour former un système complexe dont les interactions sont nombreuses. Sans revenir sur toutes les structures et temporalités qui s'expriment au travers de ce système, il faudra néanmoins nous attarder plus précisément sur une notion le composant : la demande citadine. En effet, la demande de logement seule - entendue uniquement dans un sens quantitatif et reliée directement au marché de l'immobilier - ne suffit pas à expliquer la gentrification. La croissance des ménages, les transformations économiques, les politiques successives de logement - informations mesurables quantitativement - ont certes bouleversé les mentalités, changé les habitudes des résidents, transformé les espaces urbains centraux; mais elles ne justifient pas à elles seules une volonté affichée, un véritable engouement de citadinité et de nouvelles centralités ${ }^{6}$ pour certaines personnes : un choix qualitatif se pose pour les ménages solvables vis-à-vis de leur future résidence et de son emplacement dans un quartier central ou péricentral. Le confort du logement choisi n'est plus suffisant ; l'achat ou la location d'une résidence principale suppose un espace environnant urbain riche en activités ou en équipements, dans un cadre agréable. « La nature des aspirations s'est déplacée : satisfaire une fonction de base comme se loger ne suffit plus, il faut qu'elle soit satisfaite dans les meilleures conditions de qualité ${ }^{7}$. Ces ménages créent ainsi une véritable nouvelle demande citadine, à laquelle le centre d'une ville peut répondre, et que nous allons approfondir.

Après un bref exposé des causes principales socio-économiques de la mise en place de la gentrification et de la demande sociale citadine, nous aborderons cette problématique spécifique en partant d'un exemple concret, Strasbourg, et plus particulièrement de son centre. Une enquête, effectuée en 1997 sur cette base géographique, permettra d'affiner la recherche.

\section{Gentrification et demande socio-citadine}

4 La description des différentes tendances sociales générales, structurant les agglomérations, permet d'aborder le cœur du sujet, la gentrification et la demande sociale citadine sous-jacente, sachant que la demande, prise dans le sens global, détermine avant tout "l'action de faire savoir ce que l'on souhaite obtenir" (Larousse). Cela se fera en fonction du « on », en l'occurrence de la société. Or, si celleci change, les demandes changent aussi.

\section{A. De nouvelles structures éco-démographiques}

5 Pour les pays industrialisés, la dévalorisation du secteur industriel est un phénomène d'ordre mondial. La productivité du secteur secondaire augmente très rapidement après la Deuxième Guerre mondiale, entraînant une baisse des actifs. Avec l'élargissement systématique de la division du travail à l'ensemble des secteurs industriels, la « grande firme » ne conserve guère d'implantations stables et créatrices 
d'emplois, essentiellement qualifiés, que dans certains secteurs géographiques des capitales et des métropoles : couronnes externes des grandes régions urbaines de Paris et de Londres, ou des villes de la Randstadt des Pays-Bas, des mégalopoles américaines

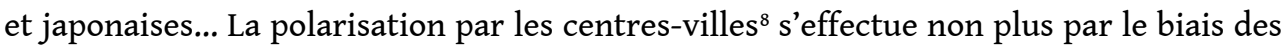
industries manufacturières mais par le développement des emplois tertiaires. Déjà, au niveau de l'architecture, l'esthétique urbaine par exemple, le bâtiment administratif est différent de l'usine: il fait davantage partie intégrante de la ville. De plus, d'une manière générale, les salaires du tertiaire sont plus élevés car la qualification demandée est supérieure ${ }^{9}$ à celle du secondaire ; tout ceci favorise la consommation.

6 La contradiction principale des rapports que le tertiaire urbain entretient avec l'espace réside dans le fait qu'il tend conjointement à se concentrer en quelques points nodaux de la ville et à se diffuser dans l'ensemble du tissu urbain ${ }^{10}$. Des métropoles, ne comptant que quelques centaines de milliers d'habitants, voire des villes moyennes, se sont dotées, dans les années 1970-1980, de centres culturels, commerciaux ou de direction, souvent édifiés avec le concours de la puissance publique. En France, la PartDieu à Lyon, Mériadeck à Bordeaux... témoignent de ce dynamisme. Signalons pourtant que le développement de cette tertiarisation est à nuancer. Bien que cela puisse paraître paradoxal, "les services vont moins bien quand l'appareil de production se détériore... On croyait à la substitution, on découvre la ségrégation $»^{11}$. Il y a des effets d'interaction entre les deux secteurs, le processus n'est donc pas univoque.

7 Dans un monde en équilibre dynamique, les changements démographiques tendent à accentuer ces mutations socio-spatiales. Dans certaines cités déclinantes, notamment leur centre, le nombre de ménages a augmenté alors que la « crise des centres ${ }^{12}$ se fait sentir. Par exemple, à Pittsburgh (États-Unis), entre 1970-1980, la population a baissé de $5,7 \%$ alors que les ménages s'accroissent de 9,1\%. La situation en Europe est identique. La demande en logement change, la priorité se portant vers les studios et les deux-pièces, et se renforce avec la présence d'une proportion grandissante de célibataires. Celle-ci apparaît dans les statistiques concernant les caractéristiques des mariages. En France, en 1994, l'âge moyen au premier mariage s'élève à 27,3 ans pour les femmes (22,4 ans en 1970) et à 29,2 ans pour les hommes (24,4 en 1970). En outre, les mariages se raréfient : le nombre maximum avait atteint 417000 en 1972. La baisse a ensuite été continue pendant 15 ans, alors que le nombre de personnes en âge de se marier augmentait (conséquence du «baby-boom »). Ainsi, le taux de nuptialité a chuté dans des proportions importantes : 4,4\%o en 1996 (soit 279000 unions célébrées) contre $8,1 \%$ en 1972 , bien que la tendance s'inverse depuis 1988. Mais il ne faut pas oublier que le nombre de divorces continue de croître ${ }^{13}$.

Dès lors, la demande de logements et les conditions de vie diffèrent. Bien qu'il ne faille pas tomber dans un déterminisme démographique, il est certain que cet " ajournement » du mariage n'empêche guère les unions libres, mais retarde tout de même la venue des enfants. Par ailleurs, la femme, du fait de la tertiarisation, a plus de possibilités de trouver un emploi que dans les années 1940-1950; elle aura plus d'opportunités de travail, plus d'indépendance, plus de liberté. Or, si un couple a des enfants, cela implique un engagement financier substantiel dans les dépenses liées au logement et dans les activités liées à la famille proprement dite. Ceux qui n'ont pas opté pour cet engagement souhaiteront se divertir davantage, auront plus de relations endehors de leur quartier, etc. Ces activités s'effectuent aisément si l'on habite le centreville qui concentre nombre d'équipements divers et variés. Dès la fin des années 60 , une 
demande plus exigeante, plus spécialisée, caractérisée par un usage grandissant des activités proposées par la vie citadine, se profile dans l'environnement urbain.

\section{B. Vers une nouvelle volonté sociale citadine?}

9 «Les modes de vie et les styles de vie sont des concepts évolutifs qui suivent l'évolution de la société. Ils se traduisent par des conceptions qui varient selon l'âge et la situation professionnelle ou familiale des personnes mais aussi selon les lieux et les époques ${ }^{14}$. Un jeune couple, sans enfant, qui entre dans la vie active, aspire à des occupations autres qu'une famille ayant des enfants. Il cherche une certaine animation, nocturne ou diurne, que peut lui procurer le centre-ville, et en même temps la proximité de l'emploi. Toutes ces données alimentent automatiquement des différenciations dans les choix résidentiels. A. Warde ${ }^{15} s^{\prime}$ attache ainsi aux caractéristiques socioprofessionnelles des fractions de la "classe moyenne" qui y participe; l'emploi des femmes dans les fonctions d'encadrement semble bien refléter cette volonté sociale. En résumé, dans le choix «théorique » d'un quartier, la famille tient compte essentiellement des structures du logement (espace, confort) et de son prix, des aspects socio-économiques qui semblent se refléter dans le quartier (classes, composition ethnique) et des données pratiques le composant (accessibilité, chalandise...). Eu égard à l'importance des critères cités, chaque ménage a son propre classement, qui varie selon sa place dans le cycle de vie, son statut socio-économique... Grâce à un salaire plus élevé, élargissant de facto les possibilités quant au choix de la résidence et de sa localisation, la famille ou le couple sera attiré par des groupes d'immeubles offrant de nombreux choix de services ou des agréments largement variés dans le quartier comme les parcs, une belle architecture, des musées, des universités ou une bonne distribution commerciale, formant une zone de chalandise concentrée et néanmoins variée, incluant également des divertissements ou enfin une accessibilité optimisée par les rocades et pénétrantes autoroutières.

Ces types de quartiers se situent généralement dans les centres de villes. Malgré l'apparente ou réelle vétusté de bon nombre de leurs îlots ${ }^{16}$, dans les années 60 , voire 70 , ces secteurs ont conservé leurs potentialités ${ }^{17}$ grâce à leur situation centrale, privilégiée. Un étudiant, célibataire et sans enfant, a tendance à se rapprocher et de son université et du centre-ville car, là, prédomine une ambiance dynamique et entraînante. Ceci ne peut se faire que si son budget le lui permet.

11 Ainsi se développe une nouvelle citadinité, caractérisée essentiellement par de jeunes ménages aux revenus moyens ou élevés, bien intégrés dans la société, grâce notamment à leur niveau d'études : le cœur urbain, en (re)devenant un lieu d'attraction, traduit la transcription spatiale de cette citadinité. En effet, en dépit de la "crise des centres", accompagnée cependant d'un accroissement non négligeable du pouvoir central de décision, des structurations différentes interviennent, des volontés citadines se mettent en place; les offres résidentielles apparaissent à nouveau attractives pour les habitants: la gentrification est en marche. Ces nouvelles centralités, façonnées au départ grâce à une historicité ${ }^{18}$ et un symbolisme accrus (prestige du centre, bon vivre, patrimoine...), ne répondent pas aux modèles économiques classiques de localisation, mais à une demande où les images et les significations tiennent une place essentielle ; " peu importe ce qui se passe : cela se passe ici ${ }^{19}$ renchérissait un habitant de Chicago. Effectivement, les rues piétonnes se multiplient, les transports en commun se développent. La ville n'est plus seulement active, elle devient également festive ${ }^{20}$. Les 
sensibilités et les goûts du public changent, les mouvements résidentiels s'intensifient : le centre attire à nouveau, autrement.

Bien que le renforcement de la centralité se soit donc, dans un premier temps, effectué aux dépens du secteur résidentiel par des restructurations économiques, le changement des données sociales a provoqué dans un second temps, très proche, l'envie d'améliorer cette partie centrale pour le bien-être de la ville entière, provoquant un mouvement de gentrification significatif. Nous passons ainsi d'une évolution socio-économique à une demande sociodémographique, fortement accentuée par une volonté citadine. Cette vision "qualitative ", complémentaire de la démarche théorique quantitative, suggère une approche différente de la ville. En effet, des désirs d'activités culturelles et/ou de loisirs plus nombreux, des souhaits d'un regain d'équipements plus variés dans les centres-villes s'établissent et marquent cette recherche de nouvelles centralités. Les personnes-acteurs de la gentrification nourrissent cette recentralisation en souhaitant toujours davantage de fonctions qui entraînent, par effet rétroactif, une accélération du processus de gentrification. C'est à l'échelle locale que nous allons examiner cette volonté.

\section{L'exemple d'un centre-ville, Strasbourg}

La métropole alsacienne a connu de nombreux changements qui reflètent relativement bien, dans leur singularité, les différentes mutations sociales, politiques et économiques intervenues en France depuis ces dernières décennies. Il est ainsi possible de la prendre comme exemple pour illustrer le processus de gentrification. En outre, la familiarité du terrain d'étude donne l'opportunité de saisir concrètement la demande citadine en privilégiant l'échelle microsociale, l'individu. Cette pratique suppose l'élaboration d'une enquête à partir d'une problématique bien déterminée.

\section{A. Un objet géographique délimité}

Étant donné que la gentrification a comme assise territoriale les centres de villes, nous privilégions dans cette étude un centre urbain strasbourgeois élargi (fig. 1). 
Figure 1 : Cartes de localisation

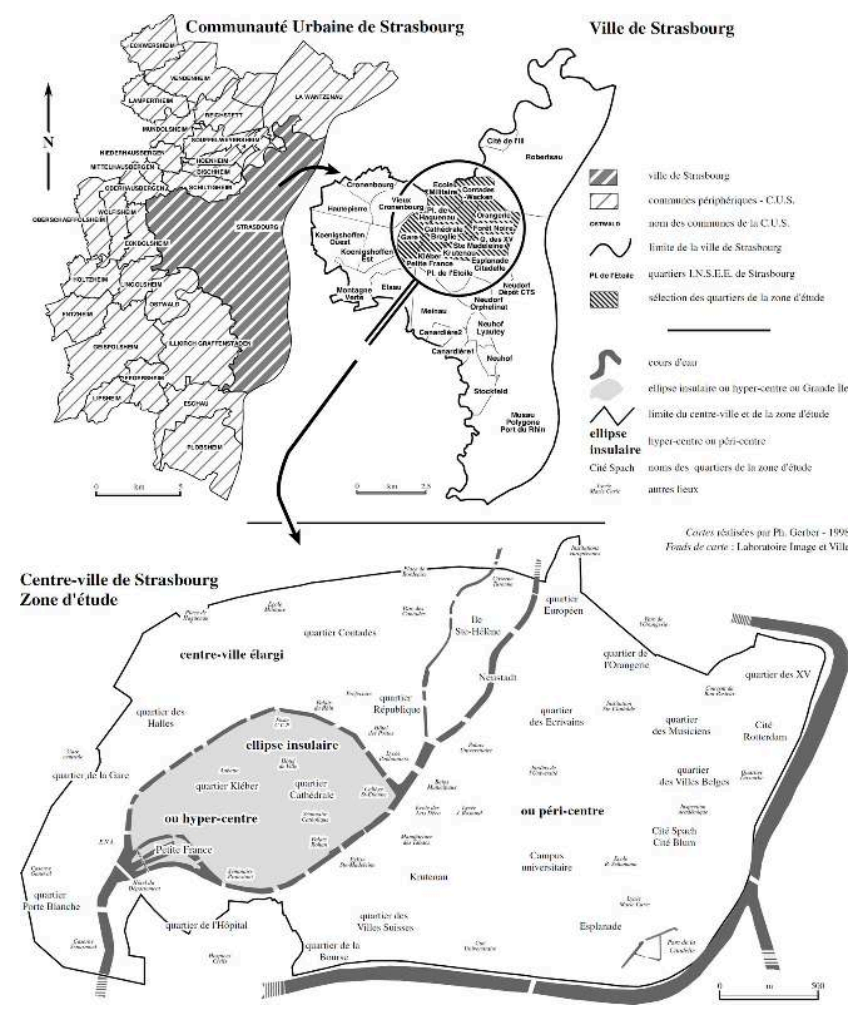

15 Cette partie de Strasbourg exclut les faubourgs extérieurs, comme la Robertsau ou le Neudorf. La délimitation ${ }^{21}$ correspond en réalité aux étapes morphologico-historiques du développement de la ville, depuis l'époque romaine jusqu'au début du XXe siècle (fig. 2).

Figure 2 : Historique de la croissance spatiale de Strasbourg intra-muros

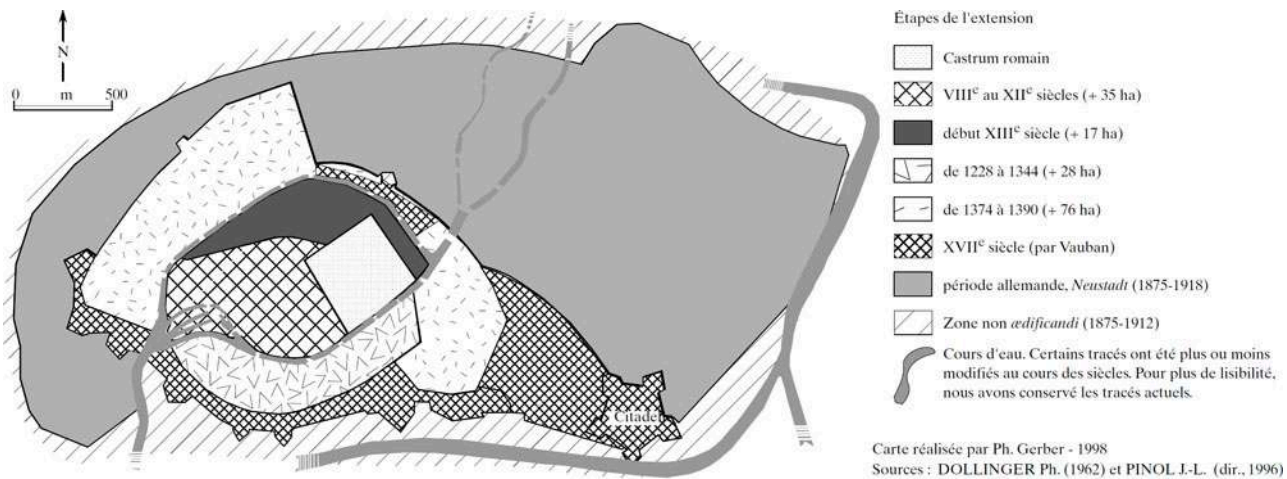

Les opérations concernant plus particulièrement le processus de gentrification, commencent, quant à elles, dès les années 1950 et privilégient nettement un urbanisme fonctionnaliste et progressiste, caractéristique de l'époque.

La période de recentralisation s'étale sur 25 ans environ et débute officiellement par la loi-cadre du 31 décembre 1958, correspondant à la politique nationale de la Rénovation Urbaine. Elle se concrétise, à Strasbourg, par un redéploiement de l'hypercentre ${ }^{22}$ grâce à différents travaux: a) l'aménagement des Halles, fin des années 70, avec un programme de bureaux, commerces, hôtels, et d'importants parkings. L'opération bénéficie d'avantages indéniables, notamment du point de vue de l'accessibilité: 
rocades autoroutières, parkings, stations de bus; $b$ ) le choix de la ville comme siège du Conseil de l'Europe dès 1949, dans un cadre prestigieux, près de l'Orangerie, au nord de la zone d'étude. La ville partage ainsi, avec New York et Genève, le privilège d'être le siège d'organisations internationales sans être capitale d'État, ce qui lui donne sans conteste une nouvelle centralité internationale ; c) un aménagement structurant de transport public, le tramway, conçu en site propre; l'idée date de 1970, mais l'inauguration n'eut lieu qu'en 1994. On découvre, le long de son parcours, de grandes places où s'affairent piétons et cyclistes. Le réaménagement de ces lieux touche également des axes qui relient deux secteurs historiques, piétonniers depuis plus de vingt ans: le quartier de la Cathédrale (1972) et celui de la Petite France (1977); d) l'édification en 1960, dans une zone péricentrale, du grand quartier résidentiel de l'Esplanade, que jouxte le nouveau campus universitaire ; enfin, e) d'autres opérations de démolition-reconstruction (Rénovation Urbaine) furent entreprises dans la vieille ville, entraînant l'expulsion de ménages modestes et favorisant timidement la gentrification. La population du centre passe de 97768 habitants en 1968 à 84852 en 1982, soit une perte de près de 13000 personnes.

On assiste dans les années 1970 à un revirement. La mise en place, en 1974, d'un secteur sauvegardé (restauration) marque la transition entre une politique de recentralisation/ rénovation et une politique de revitalisation/réhabilitation. Les habitations ne sont plus démolies pour être remplacées, mais restructurées selon des règles d'urbanisme : "Les travaux visent à introduire les normes de confort et d'habitabilité, à remettre en état le gros cuvre en conservant le caractère des façades et éventuellement des cages d'escalier, etc. $»^{23}$. Des aides de l'État compensent le coût des investissements nécessaires pour ces travaux, dans le but de maintenir la population résidante, ce qui n'était pas le cas lors des opérations de rénovation. Ces réhabilitations peuvent se développer ponctuellement ou prendre la forme d'O.P.A.H. ${ }^{24}$, fixant un périmètre sur lequel l'A.N.A.H. ${ }^{25}$ intervient. Certains propriétaires-bailleurs saisissent d'ailleurs l'occasion de ces embellissements du voisinage pour effectuer ce type de travaux afin d'augmenter la plus-value de leur patrimoine. Trois O.P.A.H. ont été réalisées. La première se situe à la Krutenau (1978 à 1980), touchant environ 820 logements. Suivront celle de la Petite France (ellipse insulaire), entre 1982 et 1985, et enfin celle de la gare (à l'ouest), dernière en date (1988-1991). D'autres îlots seront réhabilités mais en-dehors du cadre des O.P.A.H. Le résultat principal de ces opérations conduit à stabiliser la population centrale à 84025 habitants en 1990, ce qui montre que la période de restructuration, où l'on a remarqué une baisse de 13000 habitants, n'est en rien une revitalisation ${ }^{26}$. La réhabilitation attire davantage une population composée de classes moyennes ou aisées $^{27}$, favorisant ainsi la gentrification. La répartition spatiale de la gentrification ${ }^{28}$ se cantonne à quelques secteurs, en particulier à la vieille ville (ellipse insulaire et Krutenau essentiellement) où les opérations s'étalent plus largement, prouvant par là que ce processus socio-résidentiel est hétérogène au niveau infracommunal. La Neustadt n'est en effet guère touchée par ce processus; en effet, composée d'immeubles de haut standing, elle est, depuis les origines, peuplée d'une population plutôt bourgeoise.

19 Ces politiques urbaines successives, ainsi que le suivi des investissements privés, ne suffisent pourtant pas à rendre compte du processus de gentrification. Un autre facteur intervient, qui est la demande citadine. 


\section{B. Une enquête d'analyse microsociale}

Les souhaits des résidants centraux demeurent nombreux. Pour les saisir, une enquête s'avère indispensable. En partant de l'hypothèse que la gentrification est renforcée par une demande sociale de plus en plus citadine, l'enquête vise à déterminer cette demande. En suivant A.A. Moles et E. Rohmer, plusieurs paramètres, liés entre eux, devraient figurer dans l'enquête engagée : l'homme possède en effet ses «zones qui s'éloignent peu à peu de lui comme point de référence, et dont il vit une typologie : [...] les coquilles de l'homme ${ }^{29}$. En distinguant dans ces coquilles les trois qui nous intéressent, le logement, le voisinage/quartier et le centre-ville, un schéma explicatif se constitue qu'il est possible d'illustrer comme suit (fig. 3).

Figure 3 : Thèmes abordés par l'enquête
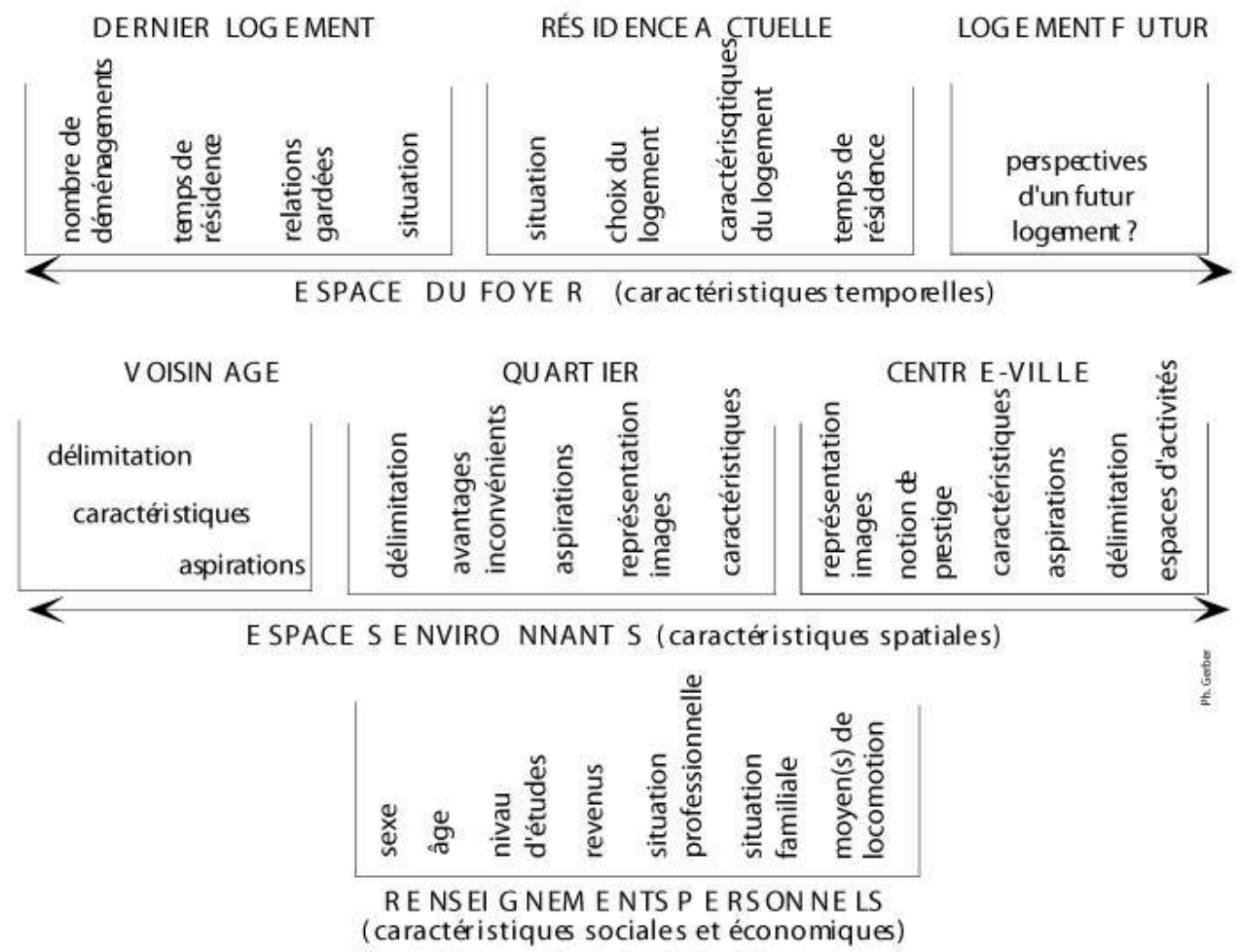

21 Les causes des mutations socio-résidentielles centrales passent par l'analyse des coquilles présentées selon des thèmes précis et particuliers ${ }^{30}$. Ainsi, les raisons qui ont poussé les habitants des quartiers du centre-ville à venir s'y installer, à y rester ou à vouloir en partir (ou non), dans un futur plus ou moins proche, sont identifiées grâce à la description spatio-temporelle du foyer qui offre le premier espace d'interface entre l'extérieur et l'intimité : il s'agit des aspects extérieurs, du choix du logement actuel, en prenant soin d'avoir une vision diachronique quant à son processus d'acquisition. Viennent, après la résidence principale, les espaces la ceinturant: le voisinage, le quartier et enfin le centre-ville. Plusieurs informations sont nécessaires à ce niveau : définitions et délimitations de ces espaces environnants, constatations, regrets ou demandes que les ménages énoncent vis-à-vis du centre.

Certaines caractéristiques sociales et économiques, comme la situation professionnelle du chef de ménage, son niveau d'études, déterminent le type de population que l'on 
interroge. Devant la masse des éléments d'information de la demande socio-citadine à recueillir, le type d'enquête retenu est le questionnaire.

La population-mère est constituée par le nombre de ménages occupant la zone délimitée. D'après le recensement le plus récent (1990), qui sert de support de travail, il existe 41139 ménages pour 84025 habitants sur le secteur étudié. Bien entendu, on ne peut pas prendre en considération toutes les personnes ou ménages y résidant : un échantillonnage représentatif stratifié, selon une logique socio-spatiale (fig. 4), est alors nécessaire.

Figure 4 : Centre-ville de Strasbourg. Localisation des rues enquêtées

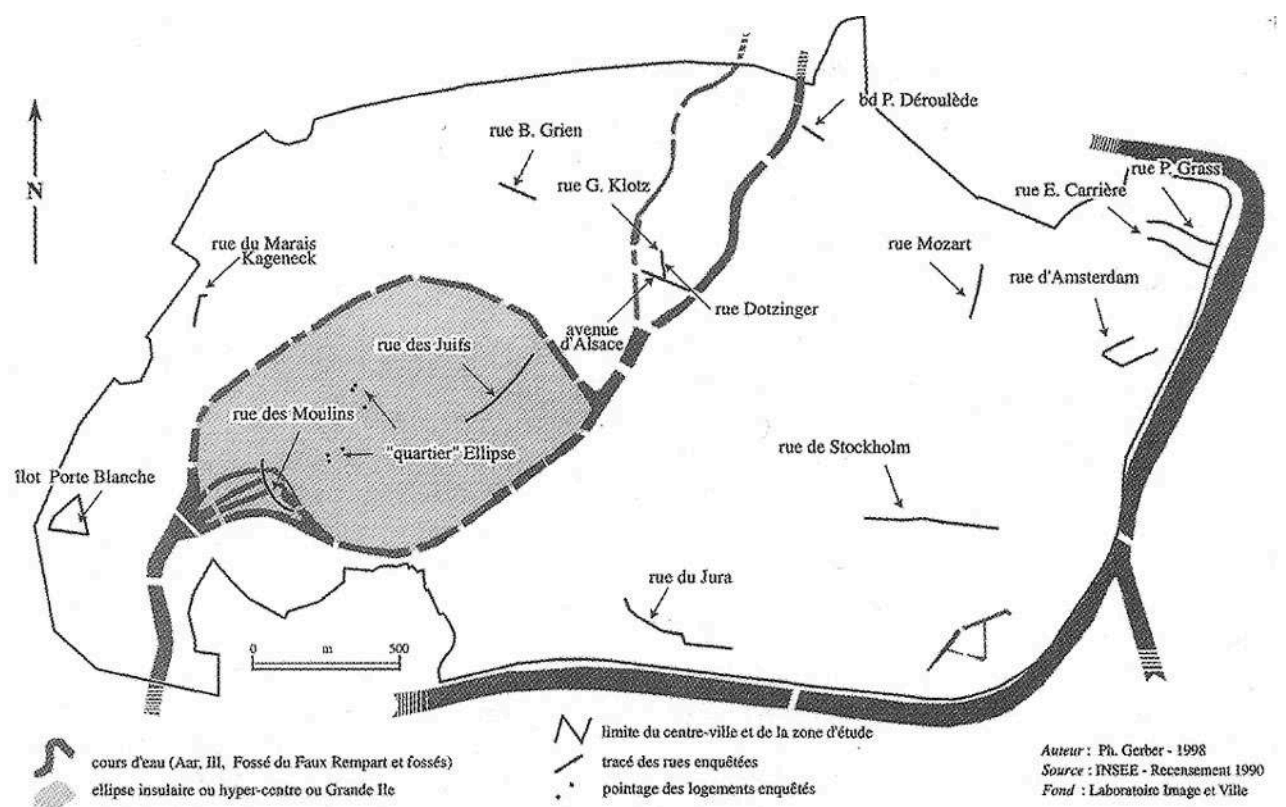

Après passation du questionnaire dans les rues indiquées sur la figure, l'échantillon de 171 enquêtes se répartit plus ou moins également sur 13 quartiers (tableau 1). 
Tableau 1 : Répartition spatiale des ménages interrogés

\begin{tabular}{|l|c|c|}
\hline \multirow{2}{*}{ Quartier } & \multicolumn{2}{|c|}{ Échantillons } \\
\cline { 2 - 3 } & Effectif brut & Proportion (\%) \\
\hline Quartier Esplanade & 23 & 13,5 \\
Quartier des Musiciens (Neustadt) & 14 & 8,2 \\
Quartier des XV (Neustadt) & 15 & 8,8 \\
Quartier Porte Blanche & 26 & 15,2 \\
Quartier Villes Suisses & 11 & 6,4 \\
Quartier Gare & 13 & 7,6 \\
Quartier Contades (Neustadt) & 9 & 5,3 \\
Quartier lle Sainte-Hélène (Neustadt) & 11 & 6,4 \\
Quartier Cathédrale & 16 & 9,4 \\
Quartier Petite France & 10 & 5,8 \\
Quartier Cité Rotterdam & 7 & 4,1 \\
Quartier de I'Europe (Neustadt) & 10 & 5,8 \\
Quartier « Ellipse » & 6 & 3,5 \\
\hline Moyenne & 13,8 & 8,3 par quartier \\
\hline Total & 171 & 100 \\
\hline
\end{tabular}

Les réponses s'avèrent variées et nombreuses selon les personnes. Résumer sans appauvrir les informations obtenues est un des buts du dépouillement et du traitement. Les variables à traiter étant mixtes - nominales ou quantitatives -, une méthode a été retenue, l'analyse des correspondances multiples (ACM), analyse factorielle qui, en ramenant toutes les informations à un niveau de mesure nominal, permet de traiter des données diverses et de prendre en compte des relations non linéaires sous-jacentes.

\section{La demande citadine. Logiques de répartition socio-spatiale}

En partant du principe qu'il existe une gentrification partielle et hétérogène à Strasbourg, il s'agit maintenant de comprendre comment la demande citadine s'introduit dans les processus de stratégies résidentielles centrales. Plusieurs classifications hiérarchiques, après ACM, ont été retenues pour indiquer les grandes structures socio-éco-spatiales. La fig. 5 apporte certains renseignements quant à la démarche choisie afin de maximiser le degré d'explication de l'échantillon et de ses descripteurs. 
Figure 5 : Analyses des correspondances multiples et classifications. Démarche et étapes suivies.

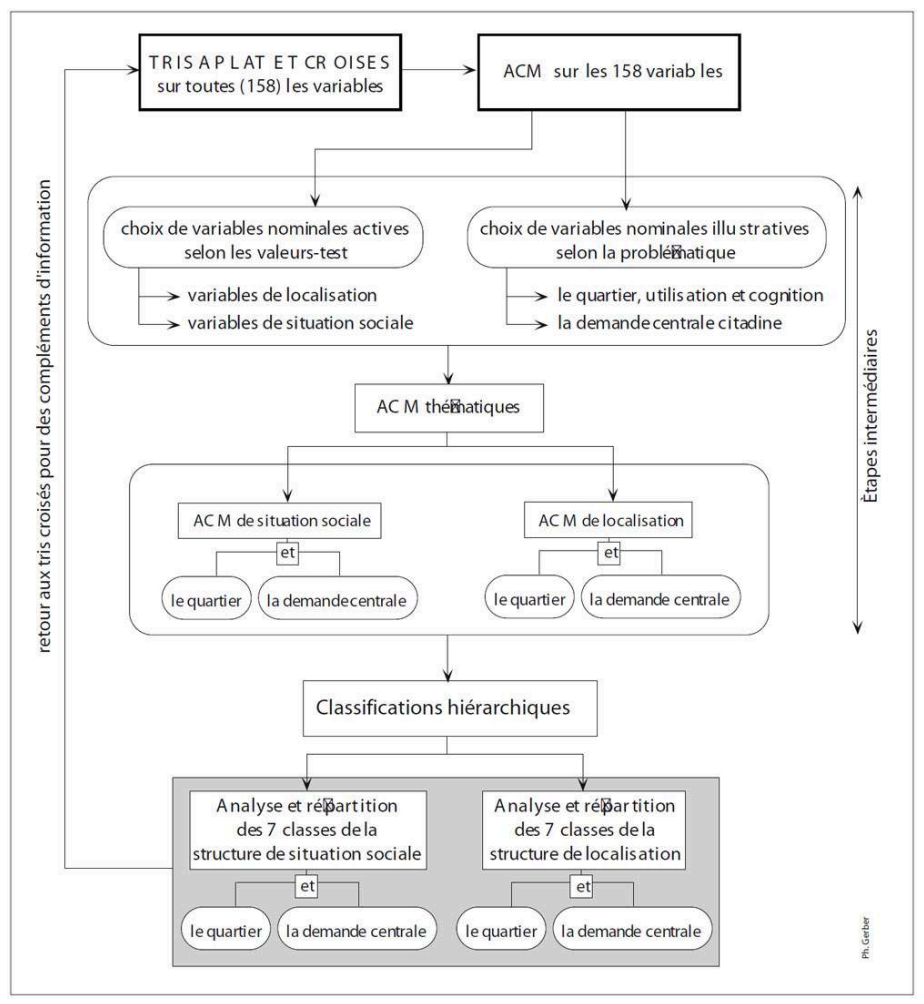

Après l'ACM générale portant sur 158 variables et plusieurs tentatives de restructuration de la matrice se dégagent les deux groupes les plus explicatifs, bien distincts. Il s'agit d'une part des variables de localisation (situation du quartier, critères de logement, années d'habitation...) et d'autre part des caractères de situation sociale, comme la catégorie socioprofessionnelle, le niveau d'études, la situation familiale.

\section{A. Impacts de la structure résidentielle sur la demande citadine}

La logique de répartition spatiale, par association des variables actives de critères résidentiels, dévoile certains groupes homogènes qui, a priori, n'étaient pas sensés se former. Ainsi se rassemblent, avec des pourcentages divers, la Petite France (secteur réhabilité devenu un haut lieu touristique) et la rue d'Amsterdam (cité péricentrale non réhabilitée), l'Esplanade et le quartier des Villes Suisses, et deux autres classes complexes : les zones Musiciens/Contades/Cathédrale et les secteurs Conseil des XV/ Cathédrale/Ellipse. La dichotomie Neustadt/Ellipse insulaire s'estompe, témoignant d'une gentrification: les quartiers s'homogénéisent. Par contre, les zones internes à la Grande Île (Cathédrale, Petite France et Ellipse) ne se regroupent pas, montrant par là qu'une généralisation doit s'effectuer prudemment. Cependant, certaines associations attendues se vérifient quantitativement comme celles des quartiers Gare et Villes Suisses ou encore l'île Sainte-Hélène (Neustadt) et la rue Baldung Grien (quartier Contades), zones plus ou moins cossues.

Pourtant, ces groupements ne parviennent pas à expliciter davantage la demande centrale. Pour pallier ces difficultés, certaines variables ont été extraites des tris croisés (tableau 2). 
Tableau 2 : La demande centrale ( en équipements) en fonction des secteurs enquêtés (en \%)

\begin{tabular}{|l|c|c|c|c|c|c|}
\hline \multirow{2}{*}{ Secteurs } & \multicolumn{3}{|c|}{$\begin{array}{c}\text { Installation bénéfique } \\
\text { commerces/services }\end{array}$} & \multicolumn{3}{c|}{$\begin{array}{c}\text { Installation bénéfique } \\
\text { loisirs/cultures }\end{array}$} \\
\cline { 2 - 7 } & Oui & Non & Sans objet & Oui & Non & Sans objet \\
\hline Esplanade (rue de Stockholm) & 21,8 & 65,2 & 13 & 29,2 & 56,5 & 14,3 \\
\hline Musiciens (rue Mozart) & 50 & 28,6 & 21,4 & 28,6 & 64,3 & 7,1 \\
\hline $\begin{array}{l}\text { Conseil des XV } \\
\text { (rues Grass et Carrière) }\end{array}$ & 41,1 & 53,3 & 6,6 & 47,7 & 53,3 & 0 \\
\hline Porte Blanche & 49 & 57,7 & 4,3 & 42,4 & 46,1 & 11,5 \\
\hline Villes Suisses (rue du Jura) & 8,8 & 81,1 & 9,1 & 90,9 & 9,1 & 0 \\
\hline Gare (rue du Marais Kageneck) & 30,8 & 61,5 & 7,7 & 61,5 & 23,1 & 15,4 \\
\hline Contades (rue Baldung Grien) & 34,4 & 55,5 & 11,1 & 67,7 & 33,3 & 0 \\
\hline $\begin{array}{l}\text { lle Saint-Hélène } \\
\text { (rues Klotz, Dotzinger, Alsace) }\end{array}$ & 45,3 & 45,6 & 9,1 & 36,4 & 54,5 & 9,1 \\
\hline Cathédrale (rue des Juifs) & 68,7 & 31,3 & 0 & 43,7 & 43,8 & 12,5 \\
\hline Petite France (rue des Moulins) & 10 & 80 & 10 & 67,7 & 33,3 & 0 \\
\hline Cité Rotterdam (rue d'Amsterdam) & 57,1 & 28,6 & 14,3 & 57,1 & 28,6 & 14,3 \\
\hline Europe (bd P. Déroulède) & 50 & 50 & 0 & 50 & 50 & 0 \\
\hline Ellipse & 50 & 50 & 0 & 50 & 50 & 0 \\
\hline Moyenne & 39,8 & 52,9 & 8,2 & 51,8 & 41,9 & 6,5 \\
\hline
\end{tabular}

31 Le croisement de ces variables apporte un complément d'information en révélant des particularités en relation avec certains quartiers étudiés. Ainsi, plus de $80 \%$ des personnes du secteur des Villes Suisses remarquent qu'il existe suffisamment de commerces/services dans le centre tandis que $91 \%$ souhaitent une plus grande offre d'équipements de loisirs/culture. Le quartier Gare, associé par la classification au secteur Villes Suisses, présente des caractéristiques similaires mais dans des proportions moindres. Par contre, le groupe Ste-Hélène/Contades n'offre pas la même homogénéité en ce qui concerne la fonction culturelle : le premier quartier souhaiterait à $67,7 \%$ davantage d'activités tandis que le second n'arrive qu'à $36,4 \%$. Et, comme nous l'avons mentionné, opérer une association entre deux quartiers de la Grande Île paraît dangereux : le secteur de la Cathédrale "désirerait» davantage de commerces alors que la Petite France est le quartier, avec celui des Villes Suisses, qui «n'accepterait» pas une augmentation des vitrines.

Toutefois, ces constatations ne remettent pas en cause la classification et son pouvoir structurant. Cette technique ouvre au contraire une voie d'explication, en ce sens qu'il n'existe pas obligatoirement un lien direct et précis entre la localisation intra-urbaine centrale des habitants et leur demande en équipement. Cela révèle, à cette échelle du moins, le caractère a-spatial de la demande citadine centrale.

Le bâti résidentiel s'avère idéal pour rendre compte de l'homogénéisation du centreville vers une gentrification, mais il reste imprécis par rapport à l'hypothèse soulevée. Une référence spatiale, précise, concrétisée ici par les variables actives de localisation, peut répondre à une question de contexte spatial, comme l'évolution du quartier, les avantages et inconvénients du voisinage, mais ne saurait satisfaire entièrement des questions se rapportant plus à des circonstances personnelles et sociétales. Or, l'interprétation de la demande citadine se rattache davantage à cette situation.

\section{B. Rôle des structures socio-économiques}

Parmi les variables portant sur les renseignements personnels (caractéristiques des ménages, nombre d'enfants, profession), l'activité structure solidement la 
classification. En effet, sur les sept classes, la distinction est relativement explicite : a) les non-actifs se séparent en trois ensembles: les chômeurs (15 personnes), les étudiants (35) et les retraités (29). Ils rassemblent 79 individus, soit en tout $46,2 \%$ de l'échantillon; b) les actifs se partagent les quatre autres groupes: les catégories supérieures aisées divisées en deux parties - les jeunes cadres et employeurs représentent $16,4 \%$ de l'échantillon, les autres, plus âgés, ont le même pourcentage -, les professions intermédiaires (gagnant entre 100 et 250 000F par an) avec 31 individus, et enfin les ouvriers qui se détachent toutefois, malgré leur faible représentativité ( $6,4 \%$ de l'échantillon, le plus petit pourcentage).

35 Ces coupures, relativement nettes, distinguent les motivations de chaque groupe, selon une vision différente des critères de localisation. Ainsi, malgré une a-spatialité des variables actives, certaines spécificités ressortent: a) l'évolution du quartier, que l'enquêté(e) remarque, ne dépend pas de son statut économique, mais de certains aspects généraux, comme l'année d'installation dans le quartier, le statut familial et le nombre d'enfants. De plus, la majeure partie de l'échantillon considère que son quartier a globalement périclité ; b) l'utilisation du quartier pour les achats/services dépendra de la situation professionnelle : les salariés utiliseront moins le quartier que les nonsalariés et se déplaceront le plus souvent en voiture pour faire leurs achats en périphérie. Les étudiants se trouvent isolés car, sans être officiellement comptabilisés en tant qu'actifs, ils exercent néanmoins une activitéé ${ }^{31}$.

Le centre-ville est une notion que les enquêtés saisissent; ils ont des opinions à exprimer à son propos. Les résultats extraits de ces dernières variables actives prouvent une tendance à une homogénéisation intra-classes : a) les étudiants sortent $\mathrm{du}$ lot car ils réclament davantage d'équipements culturels. Ils prônent une «individualité de proximité », témoignant d'une relative indifférence quant à l'utilisation des commerces/services du centre ou du quartier; b) les retraités proposent le contraire. Ils remarquent que les commerces de proximité commencent à faire défaut. En effet, les grandes surfaces monopolisent la clientèle, entraînant la fermeture des petites épiceries, posant un problème aux personnes âgées, de plus en plus nombreuses ${ }^{32}$, qui voient leur espérance de vie s'allonger tandis que la facilité de déplacement baisse avec l'âge ; c) les classes supérieures aisées, jeunes et moins jeunes, ainsi que les professions intermédiaires, se ressemblent quant à l'appréciation qu'ils ont du centre-ville. Elles le sollicitent beaucoup pour des achats rares, elles tiennent aussi à le voir se (re)valoriser par le patrimoine pour les uns, la réhabilitation, le développement de commerces et les aménagements esthétiques pour les autres, sans que cela nuise aux banlieues. Ces citadins ${ }^{33}$ cherchent ainsi plus de centralité et participent pleinement au processus de gentrification; d) les employés jouent la carte de la mobilité. Intéressés par l'évolution du centre-ville, ils l'utilisent pourtant sans plus, maximisant leurs déplacements et leur temps d'achats; e) les autres classes, comme celle des ouvriers ou des chômeurs, sont plus hétérogènes. En effet, les premiers se désintéressent plus ou moins du centre, prenant souvent la voiture pour effectuer leurs achats en périphérie. Tous constatent cependant qu'une amélioration se constitue par le biais de la réhabilitation; ils ne rejettent pas le processus de revitalisation.

Cette demande centrale se reflète également dans la notion de prestige ${ }^{34}$ : les catégories retraités et cadres ressortent aisément à ce propos dans le tableau 3. 
Tableau 3 : La notion de prestige selon l'activité (en \%)

\begin{tabular}{|c|c|c|c|c|c|c|}
\hline \multirow[b]{3}{*}{ Activité } & \multicolumn{6}{|c|}{ Prestige souhaité ou non souhaité } \\
\hline & \multicolumn{3}{|c|}{ Souhaité } & \multicolumn{3}{|c|}{ Non souhaité } \\
\hline & $\begin{array}{c}\text { Par rapport } \\
\text { aux autres } \\
\text { villes }\end{array}$ & $\begin{array}{c}\text { Par rapport } \\
\text { aux autres } \\
\text { quartiers }\end{array}$ & $\begin{array}{c}\text { Indépen- } \\
\text { damment } \\
\text { aux } \\
\text { quartiers } \\
\text { ou aux } \\
\text { villes }\end{array}$ & $\begin{array}{c}\text { Cumul } \\
\text { des } \\
3 \text { colonnes }\end{array}$ & $\begin{array}{c}\text { Non } \\
\text { prestige }\end{array}$ & $\begin{array}{c}\text { Non } \\
\text { réponse }\end{array}$ \\
\hline Employeurs, cadres & 42 & 2 & 24 & 68 & 26 & 6 \\
\hline Cadres moyens, salariés & 30,8 & 5,1 & 20,5 & 56,4 & 43,6 & 0 \\
\hline Ouvriers & 22,2 & 0 & 22,2 & 44,4 & 33,3 & 22,2 \\
\hline Retraités & 48,5 & 0 & 24,2 & 72,7 & 18,2 & 10 \\
\hline Chômeurs & 18,8 & 6,3 & 25 & 50 & 37,5 & 12,5 \\
\hline Étudiants, appelés du contingent & 56,5 & 0 & 8,7 & 65,2 & 30,4 & 4,4 \\
\hline
\end{tabular}

Malgré la prédominance des cadres, étudiants et retraités, presque tout le monde tient à donner son avis sur le cœur urbain. Seuls les ouvriers se différencient avec plus de $22 \%$ de «non réponse ». Les retraités, dont la demande citadine n'est pas très forte, insistent davantage sur les souhaits de prestige pour le centre: les sensibilités culturelles et historiques jouent un rôle important dans la structuration des volontés sociales.

En observant un autre croisement de variables concernant une éventuelle installation de commerces ou de structures culturelles, le classement par catégories socioprofessionnelles n'explique pas parfaitement les demandes.

Seule la colonne «loisirs/culture» ressort clairement selon les CSP : les catégories cadres et étudiants fournissent les pourcentages les plus élevés. En effet, «Plus le niveau de vie moyen sera élevé plus les équipements offerts, ainsi que les aménagements mis en place, seront fréquentés intensément et plus la consommation des services divers se maintiendra à un niveau élevé $\aleph^{35}$. Pour les commerces/services, les résultats sont mitigés. Les employeurs-cadres dominent toujours, montrant leur "soif» de centralité. Le reste des C.S.P. semble plus désorganisé: la classification décrite auparavant dégageait plus d'homogénéité que l'extrait de ces tris croisés, prouvant que l'activité professionnelle n'explique pas, à elle seule, les aspirations de chacun. Les deux méthodes utilisées restent cependant complémentaires.

Tableau 4 : La demande centrale (équipements, en \%) selon l'activité

\begin{tabular}{|l|c|c|c|c|c|c|}
\hline \multirow{2}{*}{\multicolumn{1}{|c|}{ Activité }} & \multicolumn{3}{|c|}{$\begin{array}{c}\text { Installation bénéfique } \\
\text { commerces/services }\end{array}$} & \multicolumn{3}{c|}{$\begin{array}{c}\text { Installation bénéfique } \\
\text { loisirs/cultures }\end{array}$} \\
\cline { 2 - 7 } & Oui & Non & Sans objet & Oui & Non & Sans objet \\
\hline Employeurs, cadres & 53 & 43,1 & 3,9 & 52,9 & 47,1 & 0 \\
\hline Cadres moyens, employés & 23,1 & 64,1 & 12,8 & 51,3 & 43,6 & 5,1 \\
\hline Ouvriers & 34,4 & 55,5 & 11,1 & 45,5 & 33,3 & 22,2 \\
\hline Retraités & 33,3 & 57,6 & 9,1 & 42,4 & 45,5 & 12,1 \\
\hline Chômeurs & 37,4 & 56,3 & 6,3 & 37,5 & 50 & 12,5 \\
\hline Étudiants, appelés du contingent & 39,1 & 52,2 & 8,7 & 67,3 & 30,4 & 2,3 \\
\hline
\end{tabular}

\section{Conclusion}

Les politiques mises en place durant ces 40 dernières années, autant la Rénovation urbaine que la réhabilitation, entre autres, ont largement concouru à déclencher le 
processus de gentrification. Pourtant, les aspects socio-résidentiels - au niveau de l'offre/demande -, de même que les processus macro-économiques ou démographiques, ne suffisent pas à expliquer ce phénomène. Les citadins manifestent certains choix, certaines volontés urbaines qui entraînent obligatoirement une vision différente des stratégies résidentielles. Il ne s'agit plus de prendre uniquement en considération la demande de logement en terme quantitatif, mais également la demande citadine, caractérisée par la recherche de (nouvelles) fonctions centrales de la part de la population centrale.

Dans cette enquête représentative du centre urbain strasbourgeois, la structuration socio-spatiale nous éclaire quant au processus de gentrification et aux aspirations citadines dans le paysage morphofonctionnel urbain. La demande centrale passe en premier lieu par l'envie de reconnaissance et de prestige du centre, par son influence, par une sorte de "volonté d'existence» établie par les individus. Ce besoin de reconnaissance est approuvé par la quasi-unanimité de l'échantillon: 63\% (108 enquêtés) estiment qu'il est nécessaire que le centre-ville de Strasbourg ait du prestige. Les raisons sont nombreuses, la principale demeurant liée au tourisme : $26 \%$ tiennent à ce que le centre attire du monde ${ }^{36}$. Les deux autres causes rejoignent la première : la zone centrale doit donner une certaine image $^{37}(24,5 \%)$ que les autres quartiers périphériques ne peuvent suivre ; en filigrane, le patrimoine constitué au centre, au fil des siècles, doit être mis en valeur pour $21,6 \%$ des personnes. D'autres raisons s'y ajoutent, mais avec des pourcentages bien plus faibles, comme la nécessité d'assurer une sécurité-propreté $(5,8 \%)$, une animation constante, un cadre de vie agréable $(10,5 \%)$. Certains pensent que le prestige est suffisant et ne doit plus être renforcé (14,6\%), alors que $10,5 \%$ souhaitent le voir véritablement augmenter.

Par contre, nous l'avions constaté lors de l'analyse des classifications hiérarchiques, les organisations de l'habitat restent moins explicites quant à la volonté citadine. En se référant au tableau 2 , les structures de résidences, du fait de leur constante homogénéisation, se prêtent difficilement à l'explication de la demande centrale citadine. En effet, la vieille ville, paupérisée et dévitalisée dès l'entre-deux-guerres, amorce, depuis le début des années 80 , une gentrification. Ce secteur se rapproche ainsi - par ses aspects socio-résidentiels - de la Neustadt, bourgeoise depuis ses origines. Quelques quartiers de ces deux entités, distinctes a priori, se rejoignent pour laisser transparaître des logiques de localisation non supposées au départ. Néanmoins, il existe une différenciation entre les îlots Cathédrale et Petite France au sein de l'ellipse insulaire, révélant une hétérogénéité interne.

Toutefois, l'autre groupe explicatif des classifications nous renseigne davantage sur les aspirations des habitants du cœur urbain. Certaines catégories sociales, notamment les étudiants et les jeunes cadres supérieurs, souhaitent davantage de fonctions centrales : qu'elles soient culturelles ou ludiques, dans une moindre mesure commerciales, les équipements mis en place renforceraient le processus de recentralisation. La gentrification favorise donc une demande centrale citadine, les acteurs étant surtout cette nouvelle population formée des jeunes classes moyennes ou aisées. En retour, la recentralisation s'effectue et attire également certaines catégories sociales susceptibles de correspondre à ce mouvement résidentiel. Gentrification et recentralisation se nourrissent mutuellement jusqu'à ne plus pouvoir marquer la distinction entre leurs causes et leurs effets, provoquant ainsi une boucle rétroactive du sous-système centreville. 


\section{NOTES}

1. Glass R. (1963), «Introduction, aspects of change », In : London, aspects of change, Centre for urban studies, London, pp. XII- XLII.

2. Baud P., Bourgeat S., Bras C. (dir., 1995), Dictionnaire de géographie, Hatier-Initial, 432 p. Brunet R. (dir., 1993), Les mots de la géographie. Dictionnaire critique, Reclus-La Documentation Française. 518 p. Gooddall B. (1987), The facts on file Dictionnary of human geography, Library of congress cataloging in publication data, U.S.A., 509 p. Johnston R.J., Gregory D., Smith D.M. (1994), The dictionnary of human geography, T.J. Press Ltd (3e édition), U.K., 723 p.

3. Gerber P. (1994), Écologie urbaine factorielle. Étude du processus de gentrification. Un essai de modélisation, mémoire de DEA de géographie, Université Louis Pasteur, Strasbourg, 156 p.

4. Les États-Unis comme l'Australie, les Pays-Bas... sous des formes et des aspects particuliers pour chaque agglomération.

5. Bailey N., Eobertson D. (1997), « Housing renewal, urban policy and gentrification », Urban studies, vol. 4, pp. 561-618. Beauregard R.A. (1990), «Trajectories of neighborhood change: the case of gentrification », Environment and planing A, vol. 22, n 7, pp. 855-874. Chevalier J., Peyon J.-P. (dir., 1994), Au centre des villes, dynamiques et recompositions, L'HarmattanGéographie Sociale, 264 p. Cortie C. \& Van Engelsdorp Gastelaars R. (1985), «Amsterdam: decaying city, gentrifying inner city?", Congrès de Norwich (G.-B.), Geo books, pp. 129-142. Friedrichs J., Dangschat J.S. (1988), Gentrification in der inneren Stadt von Hamburg. Gesellschaft für Sozialwissenschaftliche Stadtforschung, Hamburg, 115 p. Ley D. (1986), «Alternative explanations for inner-city gentrification : a canadian assessment ", Annals of the association of american geographers, vol. 76, n 4, pp. 521-535. Smith N. \& Williams P (dir., 1986), Gentrification of the city, Unwin Hyman, Boston, 257 p. La liste est loin d'être exhaustive.

6. Une centralité joue le rôle d'un pôle d'organisation de la vie sociale et économique. Il peut s'agir autant d'un centre commercial ou culturel que d'une place ou d'un monument historique, symboles représentatifs. En sachant que le centre d'une ville est constitué de plusieurs centralités, il existe parfois des hiatus entre centralités et vie sociale car une centralité peut être (inter)nationale.

7. Tobelem-Zanin C. (1995), La qualité de la vie dans les villes françaises, Publications de l'Université de Rouen, $\mathrm{n}^{\circ} 208$, p. 37.

8. En effet, «les emplacements centraux sont les plus convoités, si bien que la densité d'occupation, la valeur du sol et les prix immobiliers tendent à décroître du centre vers la périphérie. [...] Puisque cette compétition porte sur un espace polarisé par le centre-ville, elle explique en bonne partie les effets de zones concentriques qui se manifestent avec plus ou moins de netteté selon les contextes urbains" Grafmeyer Y. (1994), Sociologie urbaine, NathanUniversité, Coll. 128, p. 34.

9. Cependant un «secondaire " qualifié est d'habitude mieux payé qu'un «tertiaire » banal comme un serveur ou un pompiste. Or les «tertiaires» banals sont plus ou moins nombreux. Il faut donc se méfier des facilités de découpage sectoriel et éviter les généralisations.

10. L'emploi des verbes pronominaux « se diffuser » et « se concentrer » témoigne d'une logique de contradiction spatiale du tertiaire urbain.

11. Burgel G. (1993), La ville aujourd'hui, Hachette-Pluriel, Paris, p. 104.

12. Cf. Beaujeu-Garnier J. (1970), «Le centre des villes a-t-il encore un avenir?", Annales de géographie, $\mathrm{n}^{\circ}$ 5, pp. 494-496.

13. Source INSEE (1997), Tableaux de l'économie française, INSEE, p. 27.

14. Huet M. (1993), Les équilibres des fonctions dans la ville. Pour une meilleure qualité de vie, Rapport du Conseil économique et social, Direction des Journaux Officiels, Paris, p. 48. 
15. Warde A. (1991), "Gentrification as consumption : issues of class and gende ", Society and space, G.-B., vol. 9, $\mathrm{n}^{\circ}$ 2, pp. 223-232.

16. Les logements du quartier des Tanneurs, dans le vieux centre de Colmar, n'étaient pas équipés à $89 \%$ d'eau courante en 1968. Plusieurs résidences du Vieux Lyon présentaient les mêmes déficiences. Cf. Nonn H. et Lebeau R. In Collectif (1987). - Régions, villes et aménagement. Mélanges jubilaires offerts à Jacqueline Beaujeu-Garnier, Centre de Recherches et d'études sur Paris/Ile-de-France et Société de Géographie, pp. 373-407.

17. «Aussi la ville n'existe-t-elle en tant que potentiel d'attraction que par son centre, lieu privilégié où l'on rencontre le maximum de densité de micro-événements, lieu de la variété et de l'anonymat [...]», Moles A.A. et Rohmer E. (1972). - Psychologie de l'espace, Casterman-Poche, p. 54.

18. Cf. Bourdin A. (1984), Le patrimoine réinventé, PUF-Espace et liberté, 240 p.

19. White W.H., cité dans Moles A.A. et Rohmer E. (1972), op. cit., p. 54.

20. Burgel G. (1993), op. cit., pp. 93 et suivantes.

21. Devant les difficultés de délimiter un centre-ville, un choix a été pris a posteriori en sachant que la notion de centre historique est ambiguë bien que « les quartiers du XIXe siècle [puissent] être légitimement considérés comme historiques». Beaujeu-Garnier J., In Choay F., Merlin P. (dir., 1996), Dictionnaire de l'urbanisme, P.U.F., p. 141. Nous proposons là une délimitation d'UN centre et non DU centre de Strasbourg.

22. Cf. Badariotti D. (1994), Ville et vote. Urbanisme et géographie électorale à Strasbourg sous la Ve République, Thèse de doctorat, Université Louis Pasteur, U.F.R. de Géographie, Strasbourg, Volume 1, 266 p.

23. Merlin P. (1996), L'urbanisme, PUF-Que Sais-Je ?, p. 88.

24. Opérations programmées d'amélioration de l'habitat.

25. Agence nationale pour l'amélioration de l'habitat.

26. La revitalisation s'oppose, dans ce contexte, à la recentralisation ou « manhattanisation [qui est] un vaste mouvement de reconquête par un secteur tertiaire spécifique, où dominent des activités sophistiquées de gestion, direction et recherche » et qui laisse un centre vide de vie le soir ; Chaline C. (1980), La dynamique urbaine, PUF le géographe, p. 155.

27. Védrine H. (1979), Mieux aménager sa ville, Éd. du Moniteur, 213 p., Badariotti D. (1994). op. cit. et Merlin P. (1996). - op. cit., p. 89.

28. Cf. Cauvin C., Gerber P., en collaboration avec Bronner A.C., Hirsch J. et Schaub G. (1998), Communauté Urbaine de Strasbourg. Une première approche de la répartition socio-spatiale. État des lieux 1990, Étude réalisée à la demande de la C.U.S., Direction des Études et de la Prospective. Université Louis Pasteur, Laboratoire Image et Ville, Strasbourg, pp. 35 et suivantes.

29. Moles A.A. et Rohmer E. (1972).- op. cit., p. 41.

30. «Il est vrai qu'en s'obligeant à penser tous les enchaînements des espaces allant de l'espace de l'intimité à celui de l'agora, on se donne une chance d'imaginer des évolutions possibles, de mieux comprendre comment la ville entre dans la maison et réciproquement ». Préel B. (1994), La ville à venir, Éd. Descartes et Cie, p. 52.

31. Certains d'entre eux exercent un travail partiel en plus de l'activité estudiantine.

32. $12,4 \%$ de plus de 60 ans en 1968, 13,3\% en 1982, et $17,7 \%$ en 1990 (pour les plus de 65 ans) sur le terrain d'étude.

33. B. Préel remarque une grande attraction du centre sur les classes aisées et des jeunes dans son enquête. Les résultats principaux sont exposés dans son article «Modes de vie dans sept métropoles européennes ». In: Pumain D., Godard F. (1996), Données urbaines, Anthropos, Coll. Villes, pp. 91-99.

34. La manifestation d'un certain attrait, une certaine " grandeur » par rapport aux autres villes ou quartiers pour le centre d'une ville en général, correspond à une centralité symbolique, la notion de prestige, difficilement mesurable quantitativement. 
35. Tobelem-Zanin C. (1995), op. cit., p. 203.

36. On rejoint la pensée de la ville-musée. Cf. Mongin O. (1995), Vers la troisième ville?, Hachette, pp. 69-75 ou Bourdin A. (1984), op. cit.

37. Cette image se rattache souvent à la notion de capitale alsacienne et capitale européenne que Strasbourg doit assumer, grâce au rôle de son centre notamment.

\section{RÉSUMÉS}

Sur le marché immobilier complexe des centres-villes se greffe un processus socio-résidentiel particulier et instable, celui de la gentrification. Se plaçant dans un contexte économique et social spécifique (tertiarisation, augmentation du nombre des ménages...), ce processus nécessite cependant pour son développement un autre facteur essentiel: la demande sociale citadine, difficilement mesurable. Une étude de cas, Strasbourg et son centre urbain, permet, grâce à la réalisation d'une enquête sur le terrain, d'analyser et de comprendre cette notion.

A particularly unstable socio-residential process, gentrification, has an important influence on the complex realy estate market of city centres. Placed in a specific socio-economic context (tertiarization, increasing number of households...), the development of this process depends on one essential factor: the urban citizen's demand which is difficult to measure. A case study, of the centre of Strasbourg and a thorough inquiry permitted further understanding of this concept.

Auf den komplexen Immobilienmarkt der Stadtzentren propft sich ein besonderer und instabiler sozio-residentieller Prozess - die Gentrification. Dieser Prozess, der sich in einen spezifischen wirtschaftlichen und sozialen Kontext einfügt (Tertiärisierung, Vergrösserung der Haushaltszahl), erfordert indessen für seine Entwicklung einen anderen wesentlichen Faktor : die schwer messbare sozial bedingte städtische Nachfrage. Eine Fallstudie - Strassburg und sein Stadtzentrum - erlaubt dank der Durchführung einer Untersuchung vor Ort den Begriff der Gentrification zu analysieren und zu verstehen.

\section{INDEX}

Keywords : inner-city, recentralization, revitalization, socio-residential mutations, Strasbourg, urban functions

Schlüsselwörter : Revitalisierung, Rezentralisierung, sozialer Wohnortwechsel, städtische Funktionen, Stadtmitte, Strassburg

Mots-clés : centre-ville, fonctions urbaines, mutations socio-résidentielles, recentralisation, revitalisation, Strasbourg 


\section{AUTEUR}

\section{PHILIPPE GERBER}

Laboratoire « Image et Ville » - UPRES-A 7011 du CNRS - Université Louis-Pasteur - 3, rue de l'Argonne 67000 Strasbourg 